\title{
EDITORIAL
}

\section{STRUCTURALLY SPEAKING}

\author{
Technical advances in structural biology are beginning to help us to understand how cells work, \\ and these studies are especially pertinent to understanding microbial processes.
}

Microbiologists have traditionally exploited the simplicity of viral, bacterial and fungal systems to gain an insight into complex mechanisms and processes. And, although we recognize that microorganisms don't operate in isolation but are usually members of communities, structural biology is proving invaluable in understanding how microbial cellular processes function at the molecular level.

A quick glance at the contents pages of some key microbiology research journals reveals the ongoing impact of structural studies on microbiology. The Journal of Virology and the Journal of Bacteriology both feature sections that showcase structural information. Funding bodies in the United Kingdom, such as the Biotechnology and Biological Sciences Research Council, have set up six research centres that concentrate solely on structural biology. Similarly, there are numerous structural biology centres in the United States and worldwide.

Through a cursory examination of the premier published literature we can clearly see the impact that collaborations between microbiologists and structural biologists are having on microbiological and infectious disease research. The recent publication of X-ray crystal structures of the haemagglutinin from the 1918 influenza virus sheds new light on how avian influenza viruses might jump the species barrier and potentially cause pandemics $^{1,2}$. Only nine months after the identification of the SARS coronavirus as the causative agent of a new type of pneumonia, the structure of its main protease was solved, providing a basis for researchers to rationally design antiSARS drugs ${ }^{3}$. Reovirus, adenovirus and HIV-1 are three viruses for which collaborative teams are working towards solving the structures of all the viral proteins.

Virology is not the only discipline that has benefitted from the structural renaissance: bacterial proteins, especially surface proteins and exported proteins, such as toxins, are often ideal candidates for structural studies. The structures of all three proteins that comprise the anthrax toxin, and now small-molecule inhibitors of the toxin, have been reported ${ }^{4}$. Structural studies are now central to devising and designing new anti-infective therapies and investigating host-pathogen interactions.
Besides advances in our understanding of the microbial infection process, structural studies have also sprung some surprises in core microbiological disciplines. One study demonstrated that a quorum-sensing molecule that enables communication in some Gram-negative bacteria contains boron ${ }^{5}$. This was the first demonstration of a potential biological role for boron. Nitrogen fixation, which is restricted to bacterial and archaeal species and is crucial in global nitrogen cycling, has long been a favourite topic for study by microbial geneticists and physiologists. When the structure of bacterial nitrogenase was determined, a 'light' atom, probably nitrogen, was detected in the enzyme cofactor ${ }^{6}$. This could prove pivotal in understanding how nitrogen is fixed. Our understanding of cell division, DNA replication and transcription has similarly been revolutionized by determining the structures of some of the proteins involved.

The mechanisms by which molecular machines, such as the flagella and pili, assemble and function have occupied the time of many microbiologists. Structures of the proteins that form these machines have already had a large impact on understanding how they work; these collaborations bode well for future advances in these fields.

With this in mind, in this month's issue we publish a review article that integrates bacterial type IV pili structures with their biological functions - an article that clearly illustrates the power of relating structural information to biological function. Because microbiologists are embracing and integrating the core discipline of structural biology with traditional microbiological endeavours, we will no doubt be featuring many more articles that herald this new era of structural microbiology.

\footnotetext{
1. Gamblin, S. J. et al. The structure and receptor-binding properties of the 1918 influenza hemagglutinin. Science 303, 1838-1842 (2004).

2. Stevens, J. et al. Structure of the uncleaved human $\mathrm{H} 1$ hemagglutinin from the extinct 1918 influenza virus. Science 303, 1866-1870 (2004).

3. Yang. H. et al. The crystal structures of severe acute respiratory syndrome virus main protease and its complex with an inhibitor. Proc. Natl Acad. Sci. USA 100, 13190-13195 (2003).

4. Collier, R. J. \& Young, J. A. Anthrax toxin. Annu. Rev. Cell. Dev. Biol. 19 45-70 (2003).

5. Chen, W. et al. Structural identification of a bacterial quorum-sensing signal containing boron Nature 415, 545-549 (2002).

6. Einsle, O. et al. Nitrogenase MoFe-protein at 1.16 Å resolution: a central ligand in the FeMo-cofactor. Science 297, 1696-1700 (2002).
} 